\title{
THE DIAMETER OF AN IMMERSED RIEMANNIAN MANIFOLD WITH BOUNDED MEAN CURVATURE
}

\author{
TH. KOUFOGIORGOS and CH. BAIKOUSSIS
}

(Received 30 June 1980)

Communicated by G. Virsik

\begin{abstract}
Let $M$ be an $n$-dimensional complete Riemannian manifold with Ricci curvature bounded from below. Let $\bar{M}$ be an $N$-dimensional $(N>n)$ complete, simply connected Riemannian manifold with nonpositive sectional curvature. We shall prove in this note that if there exists an isometric immersion $\varphi$ of $M$ into $\bar{M}$ with the property that the immersed manifold is contained in a ball of radius $R$ and that the mean curvature vector $H$ of the immersion has bounded norm $\|H\|<H_{0}$, $\left(H_{0}>0\right)$ then $R>H_{0}^{-1}$.
\end{abstract}

1980 Mathematics subject classification (Amer. Math. Soc.): 53 C 40.

Generalizing a result by Aminov (1973), Hasanis and Koutroufiotis proved that if there exists an isometric immersion $\varphi: M \rightarrow E^{N}$ such that $\|H\|<H_{0}$ and $\varphi(M) \subset B_{R}$, then $R \geqslant H_{0}^{-1}$, where $H$ is the mean curvature vector of the immersion and $B_{R}$ a ball of radius $R$. In this paper we shall generalize the above result considering isometric immersions of $M$ into $\bar{M}$. To prove the main theorem we need the following

LEMMA 1. Let $M$ be a complete Riemannian manifold with Ricci curvature bounded from below. If a smooth function $f$ on $M$ has an upper bound, then for any $\varepsilon>0$ there is a point where the Laplacian $\Delta f<\varepsilon$.

This is obtained easily from Theorem A of Omori (1967). For a proof see Hasanis and Koutroufiotis (1979). 
LEMMA 2. Let $M$ be a complete, simply connected Riemannian manifold with nonpositive sectional curvature. Let $\gamma:[0,1] \rightarrow M$ be a geodesic parametrized proportional to arc length. If $X$ is a Jacobi vector field along $\gamma$ such that $X(0)=0$, then

$$
\left.\left\langle\nabla_{T} X, X\right\rangle\right\rangle\langle X, X\rangle,
$$

where $\nabla$ is the Riemannian connection and $T$ the tangent vector of $\gamma$.

Proof. We consider the function

$$
\psi=\left\langle t \nabla_{T} X-X, X\right\rangle .
$$

We have

$$
\begin{aligned}
\frac{d \psi}{d t} & =t\left\langle\nabla_{T} \nabla_{T} X, X\right\rangle+\left\langle t \nabla_{T} X-X, \nabla_{T} X\right\rangle \\
& =t\langle-R(X, T) T, X\rangle+t\left\langle\nabla_{T} X, \nabla_{T} X\right\rangle-\left\langle X, \nabla_{T} X\right\rangle \\
& >t\left\langle\nabla_{T} X, \nabla_{T} X\right\rangle-\left\langle X, \nabla_{T} X\right\rangle .
\end{aligned}
$$

Since $\left\langle X, \nabla_{T} X\right\rangle^{2} \leqslant\langle X, X\rangle\left\langle\nabla_{T} X, \nabla_{T} X\right\rangle$ we have

$$
\left.\left\langle\nabla_{T} X, \nabla_{T} X\right\rangle\right\rangle\left\langle X, \nabla_{T} X\right\rangle^{2}\langle X, X\rangle \text { for } t>0 .
$$

From (1), (2), (3) we get

$$
\frac{d \psi}{d t} \geqslant \frac{\left\langle X, \nabla_{T} X\right\rangle}{\langle X, X\rangle} \psi
$$

We put $h=\langle X, X\rangle^{1 / 2}$. It follows that

$$
\frac{1}{h} \frac{d h}{d t}=\frac{\left\langle X, \nabla_{T} X\right\rangle}{\langle X, X\rangle} .
$$

Hence (4) gives

$$
\frac{h \frac{d \psi}{d t}-\psi \frac{d h}{d t}}{h}>0 .
$$

Thus

$$
\frac{d}{d t}\left(\frac{\psi}{h}\right)>0 \text { for } t>0
$$

But $\lim _{t \rightarrow 0} \psi / h=0$ and so from (5) we have $\psi / h>0$ and hence $\psi>0$ which implies the desired inequality.

THEOREM. Let $M$ be a complete Riemannian manifold with Ricci curvature bounded from below. Let $\bar{M}$ be a complete, simply connected Riemannian manifold with nompositive sectional curvature. If there exist an isometric immersion $\varphi$ : $M \rightarrow \bar{M}$ such that $\|H\| \leqslant H_{0}=$ const $>0$ and $\varphi(M) \subset B_{R}$, then $R>H_{0}^{-1}$. 
Proof. Let $Q$ be the centre of the metric ball $B_{R}$ in $\bar{M}$. Consider a vector $X$ tangent to $M$ at $P$ and a geodesic $\beta(u)$ in $M$ such that $\beta(0)=P, \beta^{\prime}(0)=X$. Let $\sigma(t, u), 0<t<1$ be a parametrization of the unique geodesic in $\bar{M}$ from $Q$ to $\beta(u)$ (proportional to arc length). Let $T, X$ be the vector fields along $\sigma$ defined by

$$
T=\sigma_{*} \frac{\partial}{\partial t}, \quad X=\sigma_{*} \frac{\partial}{\partial u} .
$$

It follows easily from this construction that, for fixed $u, X$ is a Jacobi field along the geodesic $t \rightarrow \sigma(t, u)$ with $X(0)=0$.

Now, we consider a function $F$ on $M$ defined by

$$
F(P)=\frac{1}{2}\{d(Q, P)\}^{2},
$$

where $d(Q, P)$ is the distance from $Q$ to $P$ in $\bar{M}$. We will estimate the Hessian form $\nabla^{2} F$.

We put $f(u)=d(Q, \beta(u))=\int_{0}^{1}\|T\| d t$. We obtain easily that

$$
f^{\prime}(u)=\frac{1}{\|T\|}\langle T, X\rangle_{t=1}
$$

Since $\|T\|=f(u)$ we get $f(u) \cdot f^{\prime}(u)=\langle T, X\rangle_{1-1}$, that is

$$
F^{\prime}(u)=\langle T, X\rangle_{t=1} \text {. }
$$

If $\bar{\nabla}$ is the Riemannian connection of $\bar{M}$ then

$$
F^{\prime \prime}(u)=\left\langle\bar{\nabla}_{X} T, X\right\rangle_{t=1}+\left\langle T, \bar{\nabla}_{X} X\right\rangle_{t=1}=\left\langle\bar{\nabla}_{T} X, X\right\rangle_{t-1}+\left\langle T, \bar{\nabla}_{X} X\right\rangle_{t-1}
$$
since $[X, T]=0$.

Using the Gauss equation, the fact $F^{\prime \prime}(u)=\nabla^{2} F(X, X)$, and Lemma 2 we obtain

$$
\nabla^{2} F(X, X) \geqslant\langle X, X\rangle+\langle T, a(X, X)\rangle,
$$

where $a$ is the second fundamental form of immersion. Taking the trace of this, we have

$$
\Delta F \geqslant n+n\langle T, H\rangle .
$$

But by assumption $|\langle T, H\rangle|\left\langle R H_{0}\right.$. Hence $\left.\Delta F\right\rangle n\left(1-H_{0} R\right)$. If we had $R<H_{0}^{-1}$ then $1-H_{0} R=c>0$ and so $\Delta F>n c=$ const $>0$ but this contradicts Lemma 1 , hence $R \geqslant H_{0}^{-1}$.

COROLlary. If $M$ is a complete, simply connected Riemannian manifold with nonpositive sectional curvature, then a complete minimal submanifold of $M$, whose Ricci curvature is bound from below, is extrinsically unbounded. 


\section{References}

J. Aminov (1973), 'The exterior diameter of an immersed Riemannian manifold', Math. USSR-Sb. 21, 449-454 (AMS translation).

Th. Hasanis and D. Koutroufiotis (1979), 'Immersions of bounded mean curvature', Arc. Math. (Basel) 33, 170-171.

H. Omori (1967), 'Isometric immersions of Riemannian manifold', J. Math. Soc. Japan 19, $205-214$.

Department of Mathematics

University of Ioannina

Ioannina, Greece 